\title{
Como os fatores que influenciam o engajamento impactam as avaliações dos usuários em lojas de aplicativos móveis
}

\author{
Leonardo Vasconcelos ${ }^{1}$, Raissa Barcellos ${ }^{1}$, José Viterbo ${ }^{1}$, Flavia Bernardini ${ }^{1}$, \\ Clodis Boscarioli ${ }^{2}$, Eunice Nunes ${ }^{3}$ \\ ${ }^{1}$ Instituto de Computação - Universidade Federal Fluminense (UFF) \\ Caixa Postal 24.210-310 - Niterói - RJ - Brasil \\ ${ }^{2}$ Centro de Ciências Exatas e Tecnológicas - Universidade Estadual do Oeste do Paraná \\ (UNIOESTE) Cascavel - PR - Brasil. \\ ${ }^{3}$ Instituto de Computação \\ Universidade Federal do Mato Grosso (UFMT) - Cuiabá, MT - Brasil \\ \{lvasconcellos, viterbo, fcbernardini\}@ic.uff.br, raissabarcellos@id.uff.br, \\ boscarioliegmail.com, eunice@ufmt.br
}

\begin{abstract}
Resumo. Aplicações que usam o crowdsourcing acoplado à tecnologia móvel, são chamadas de crowdsourcing móvel (CM). Aplicativos que envolvem crowdsourcing têm uma necessidade crescente de engajamento. O nosso principal objetivo foi verificar como comentários negativos - ou reclamações -, relacionados a fatores que influenciam diretamente o engajamento, influenciam a avaliação dos usuários sobre aplicativos de crowdsourcing. Cada aplicativo foi avaliado considerando os seguintes fatores que influenciam o engajamento: usabilidade, durabilidade, controle, confiança e eficiência. Os resultados experimentais verificaram que a nossa hipótese é verdadeira para ofator de durabilidade.
\end{abstract}

\section{Introdução}

O número de usuários de tecnologia móvel vem aumentando em grande escala. Entre várias aplicações móveis que foram propostas, muitas seguem uma abordagem de crowdsourcing. Quando o crowdsourcing se utiliza de tecnologias móveis, geralmente é chamado de crowdsourcing móvel (CM). Aplicativos que envolvem crowdsourcing têm uma necessidade crescente de engajamento [Larson et al. 2013]. Para um aplicativo CM ter sucesso, ele precisa ir além do uso, é necessário que os usuários estejam engajados nele [Lehmann et al. 2012]. As avaliações dos usuários agregam muito peso para que um aplicativo permaneça no mercado [Jung et al. 2012]. Elas influenciam a popularidade e aumentam o número usuários. Além disso, essas avaliações podem fornecer informações valiosas sobre vários tópicos altamente relevantes, e os desenvolvedores podem usar esse recurso para melhor atender às expectativas dos usuários, criando maneiras de engajá-los [Di Sorbo et al. 2016]. Neste artigo, apresentamos os resultados do trabalho de [Vasconcelos et al. 2019]. Investigamos a seguinte hipótese: quanto menor o número de reclamações relacionadas aos fatores de engajamento, maior a avaliação do aplicativo. Sete aplicativos para Android foram selecionados na Google Play Store. Cada aplicativo foi avaliado considerando os seguintes fatores que influenciam o engajamento [Lalmas et al. 2014]: usabilidade, durabilidade, controle, confiança e eficiência. 


\section{Metodologia}

Para a coleta de dados, selecionamos aplicativos sobre recomendações de serviços do sistema operacional Android na Play Store. Os aplicativos selecionados foram aqueles que possuem a maior quantidade de comentários na categoria: recomendação de serviços. Após a seleção, escolhemos os dez comentários mais recentes e possíveis de análise de cada aplicativo escolhido. Interpretamos cada comentário manualmente. Apontamos todas as reclamações sobre cada fator de engajamento - considerando cada definição já fundamentada na literatura.

\section{Resultados}

Realizamos uma avaliação de teste de correlação linear usando uma matriz de correlação. Como resultado, observamos que o fator de durabilidade tem maior influência na classificação das aplicações, apresentando uma forte correlação negativa. Quanto maior o número de reclamações sobre a durabilidade, menores eram as avaliações atribuídas. Após o fator de durabilidade, os fatores de usabilidade, controle e eficiência são os que mais influenciam negativamente os valores atribuídos às classificações do aplicativo.

\section{Conclusão}

Os resultados experimentais discutidos acima confirmam que a nossa hipótese é verdadeira para o fator de durabilidade. Como limitação, este trabalho foi realizado com uma pequena amostra de comentários. Como trabalho futuro, pretendemos usar análise de opinião automatizada em uma amostra maior de dados e também usar redes sociais para verificar comentários sobre os aplicativos em diferentes plataformas.

\section{Referências}

Di Sorbo, A., Panichella, S., Alexandru, C. V., Shimagaki, J., Visaggio, C. A., Canfora, G., and Gall, H. C. (2016). What would users change in my app? summarizing app reviews for recommending software changes. In Proceedings of the 201624 th ACM SIGSOFT International Symposium on Foundations of Software Engineering, pages 499-510. ACM.

Jung, E.-Y., Baek, C., and Lee, J.-D. (2012). Product survival analysis for the app store. Marketing Letters, 23(4):929-941.

Lalmas, M., O'Brien, H., and Yom-Tov, E. (2014). Measuring user engagement. Synthesis Lectures on Information Concepts, Retrieval, and Services, 6(4):1-132.

Larson, M., Said, A., Shi, Y., Cremonesi, P., Tikk, D., Karatzoglou, A., Baltrunas, L., Geurts, J., Anguera, X., and Hopfgartner, F. (2013). Activating the crowd: exploiting user-item reciprocity for recommendation.

Lehmann, J., Lalmas, M., Yom-Tov, E., and Dupret, G. (2012). Models of user engagement. In International Conference on User Modeling, Adaptation, and Personalization, pages 164-175. Springer.

Vasconcelos, L., Barcellos, R., Viterbo, J., Bernardini, F., Boscarioli, C., and Nunes, E. (2019). How factors that influence engagement impact users' evaluations in mobile app stores. In International Conference on Human-Computer Interaction, pages 571-584. Springer. 